\title{
Estadísticas de mortalidad relacionada con la salud reproductiva. México, 1997
}

E l programa prioritario de Salud E Reproductiva comprende, entre otros aspectos, la atención materna y perinatal, así como la prevención del cáncer cervicouterino y mamario. Por tal motivo, la Dirección General de Estadística e Informática pone a disposición de los lectores, algunos de los tabulados más representativos acerca del comportamiento de la mortalidad relacionada con estos aspectos hasta el año 1997.

La información fue procesada a partir de las bases de datos de mortalidad y de nacidos vivos registrados que proporciona cada año el Instituto Nacional de Estadística, Geografía e Informática a esta Dirección, en tanto que los denominadores de población tienen como fuente las proyecciones elaboradas por el Consejo Nacional de Población.

En primer término se presentan cinco cuadros para la razón de mortalidad materna, donde puede apreciarse su tendencia y su peso relativo respecto al total de defunciones entre las mujeres en edad reproductiva, desde 1980 hasta 1997. Esto permite observar una reducción de $50 \%$ en el periodo. Los siguientes cuadros se refieren a la distribución por causas y grupos de edad, donde se destaca el papel preponderante de ciertas causas como la toxemia, cuyo peso relativo alcanzó $32.8 \%$ en el último año, con tasas elevadas en las edades extremas.
El cuadro IV, en particular, presenta la razón de mortalidad materna según sus causas más frecuentes por entidad federativa a fin de comparar la aportación, en cifras absolutas, de cada una de ellas. El último cuadro sobre defunciones maternas se refiere al comportamiento de algunas variables tomadas del certificado de defunción, con lo cual se puede tener una idea de algunos factores asociados a estas defunciones, en comparación con el total de muertes entre mujeres de 15 a 49 años. Puede observarse que $22 \%$ de las muertes ocurren en el hogar y, en consecuencia, sin atención médica previa; que casi $50 \%$ ocurren en mujeres con nula o mínima escolaridad, y que la gran mayoría (68\%) no tiene acceso a la seguridad social.

Los cuadros VI y VII presentan la tendencia de la mortalidad fetal y perinatal desde 1980 hasta 1997, de acuerdo con sus componentes por edad y su distribución por entidad federativa. A este respecto conviene destacar que, dentro del subregistro de la mortalidad, el de la fetal es el más elevado. Diversas circunstancias influyen en este problema, entre las que se encuentran el desconocimiento de la importancia del registro, así como las diferencias en los procedimientos administrativos y en los códigos civiles de las entidades, que en ocasiones permiten la inhumación sin el correspondiente cer- tificado de muerte fetal. Por tal motivo, el análisis de su comportamiento debe ser hecho con estas reservas.

Los últimos dos cuadros están dedicados a la mortalidad por cáncer cervicouterino y mamario. En ellos se presenta su tendencia para el periodo 1980-1997 y su distribución por entidad federativa para el año 1997. En ambos casos la tendencia es ascendente, sobre todo para el cáncer de mama. Su peso relativo demuestra claramente su importancia, puesto que en conjunto estos dos padecimientos son responsables de casi $30 \%$ de las defunciones por cáncer en mujeres, aunque la mayor parte corresponde al cervicouterino. Se observa además que el cáncer de mama ha duplicado su tasa e incrementado en forma más consistente su peso relativo, mientras que el cervicouterino tiende a la estabilización e incluso a la disminución en los últimos años.

$\mathrm{Al}$ interior de cada entidad se observan diferencias en los riesgos, puesto que las tasas de mortalidad por cáncer cervicouterino son más elevadas en Yucatán y Nayarit, mientras que para el de mama los primeros sitios los ocupan el Distrito Federal y Nuevo León. 


\section{Cuadro I}

\section{Tendencia de la razón de mortalidad materna. México, 1980-1997}

\begin{tabular}{lllll} 
Año & Defunciones & Razón* & Tasa $^{\ddagger}$ & Peso relativo $^{5}$ \\
1980 & 2296 & 9.5 & 15.0 & 7.7 \\
\hline 1981 & 2199 & 8.7 & 13.8 & 7.4 \\
\hline 1982 & 2166 & 9.0 & 13.1 & 7.6 \\
\hline 1983 & 2133 & 8.2 & 12.5 & 7.7 \\
\hline 1984 & 2074 & 8.3 & 11.7 & 7.7 \\
\hline 1985 & 1702 & 6.4 & 9.3 & 6.1 \\
\hline 1986 & 1681 & 6.5 & 8.9 & 6.3 \\
\hline 1987 & 1546 & 5.5 & 7.9 & 5.9 \\
\hline 1988 & 1522 & 5.8 & 7.5 & 5.8 \\
\hline 1989 & 1518 & 5.8 & 7.3 & 5.7 \\
\hline 1990 & 1477 & 5.4 & 7.0 & 5.5 \\
\hline 1991 & 1414 & 5.1 & 6.5 & 5.4 \\
\hline 1992 & 1399 & 6.3 & 5.3 \\
\hline 1993 & 1268 & 5.0 & 4.8 \\
\hline 1994 & 1409 & 4.5 & 6.0 & 5.3 \\
\hline 1995 & 1454 & 4.9 & 6.0 & 5.4 \\
\hline 1996 & 1291 & 5.3 & 5.2 & 4.8 \\
\hline 1997 & 1266 & 5.0 & 4.7
\end{tabular}

* Razón por 10000 nacidos vivos registrados. Se utiliza el término razón en lugar de lo que tradicionalmente se llamaba tasa, puesto que en estricto sentido la razón expresa la relación de las muertes maternas entre los nacidos vivos registrados

₹ Tasa por 100000 mujeres en edad fértil (15-49) años. Se redefine lo que sería una aproximación a la tasa de mortalidad materna, ya que en el denominador se incluye a las mujeres en edad fértil, lo que constituye una mejor expresión de la población en riesgo, ante la imposibilidad de conocer el número de mujeres embarazadas

$\S$ Peso relativo: porcentaje respecto al total de defunciones de mujeres de 15 a 49 años

Fuente: Instituto N acional de Estadística, Geografía e Informática (IN EG I) y Dirección General de Estadística e Informática, SSA

\section{Cuadro II}

\section{Principales causas de mortalidad materna. México, 1980 y 1997}

\begin{tabular}{|c|c|c|c|c|c|c|}
\hline \multirow[b]{2}{*}{ Causa } & \multicolumn{3}{|c|}{1980} & \multicolumn{3}{|c|}{1987} \\
\hline & No. & Razón* & $\%$ & No. & Razón* & $\%$ \\
\hline Total & 2296 & 9.5 & 100.0 & 1266 & 4.7 & 100.0 \\
\hline Aborto & 194 & 0.8 & 8.4 & 107 & 0.4 & 8.5 \\
\hline Causas obstétricas directas & 2063 & 8.5 & 89.9 & 1114 & 4.1 & 88.0 \\
\hline Hemorragia del embarazo y parto & 408 & 1.7 & 17.8 & 249 & 0.9 & 19.7 \\
\hline Toxemia del embarazo & 515 & 2.1 & 22.4 & 415 & 1.5 & 32.8 \\
\hline Infecciones del aparato genitourinario & 10 & 0.0 & 0.4 & 9 & 0.0 & 0.7 \\
\hline Parto obstruido & 11 & 0.0 & 0.5 & 13 & 0.0 & 1.0 \\
\hline Complicaciones del puerperio & 208 & 0.9 & 9.1 & 106 & 0.4 & 8.4 \\
\hline Las demás obstétricas directas & 911 & 3.8 & 39.7 & 322 & 1.2 & 25.4 \\
\hline Causas obstétricas indirectas & 38 & 0.2 & 1.7 & 45 & 0.2 & 3.6 \\
\hline azón por 10000 nacidos vivos registra & & & & & & \\
\hline
\end{tabular}




\section{Cuadro III \\ Mortalidad materna por CaUSAS SEgún grupos de edad. México, 1997}

Causa

Grupos de edad

Defunciones

\begin{tabular}{lrrrrrrrrrr} 
Total & 1266 & 7 & 135 & 265 & 255 & 255 & 244 & 102 & 3 & 5 \\
\hline Aborto & 107 & 0 & 10 & 24 & 20 & 29 & 19 & 0 & \\
\hline Causas obstétricas directas & 1114 & 6 & 118 & 232 & 227 & 214 & 218 & 96 & 3 \\
\hline Hemorragia del embarazo y parto & 249 & 1 & 21 & 50 & 51 & 44 & 64 & 18 & 0 \\
\hline Toxemia del embarazo & 415 & 4 & 46 & 90 & 75 & 88 & 77 & 33 & 2 \\
\hline Infecciones del aparato genitourinario & 9 & 0 & 1 & 3 & 5 & 0 & 0 & 0 & 0 \\
\hline Parto obstruido & 13 & 0 & 0 & 2 & 2 & 4 & 3 & 2 & 0 \\
\hline Complicaciones del puerperio & 106 & 0 & 14 & 29 & 17 & 25 & 16 & 5 & 0 \\
\hline Las demás obstétricas directas & 322 & 1 & 36 & 58 & 77 & 53 & 58 & 38 & 1 \\
\hline Causas obstétricas indirectas & 45 & 1 & 7 & 9 & 8 & 12 & 7 & 1 & 0
\end{tabular}

Razones específicas por causa y edad de la madre*

\begin{tabular}{lrrrrrrrrr} 
Total & 4.7 & 6.6 & 3.3 & 3.3 & 3.8 & 6.2 & 12.5 & 14.0 & 0.2 \\
\hline Aborto & 0.4 & 0.0 & 0.2 & 0.3 & 0.3 & 0.7 & 1.0 & 0.7 & 0.0 \\
\hline Causas obstétricas directas & 4.1 & 5.7 & 2.9 & 2.9 & 3.4 & 5.2 & 11.2 & 13.2 & 0.2 \\
\hline Hemorragia del embarazo y parto & 0.9 & 0.9 & 0.5 & 0.6 & 0.8 & 1.1 & 3.3 & 2.5 & 0.0 \\
\hline Toxemia del embarazo & 1.5 & 3.8 & 1.1 & 1.1 & 1.1 & 2.1 & 3.9 & 4.5 & 0.2 \\
\hline Infecciones del aparato genitourinario & 0.0 & 0.0 & 0.0 & 0.0 & 0.1 & 0.0 & 0.0 & 0.0 & 0.0 \\
\hline Parto obstruido & 0.0 & 0.0 & 0.0 & 0.0 & 0.0 & 0.1 & 0.2 & 0.3 & 0.0 \\
\hline Complicaciones del puerperio & 0.4 & 0.0 & 0.3 & 0.4 & 0.3 & 0.6 & 0.8 & 0.7 & 0.0 \\
\hline$\quad$ Las demás obstétricas directas & 1.2 & 0.9 & 0.9 & 0.7 & 1.2 & 1.3 & 3.0 & 5.2 & 0.1 \\
\hline Causas obstétricas indirectas & 0.2 & 0.9 & 0.2 & 0.1 & 0.1 & 0.3 & 0.4 & 0.1 & 0.0
\end{tabular}

Distribución porcentual

\begin{tabular}{lrrrrrrrrr} 
Total & 100.0 & 0.6 & 10.7 & 20.9 & 20.1 & 20.1 & 19.3 & 8.1 & 0.2 \\
\hline A borto & 100.0 & 0.0 & 9.3 & 22.4 & 18.7 & 27.1 & 17.8 & 4.7 & 0.0 \\
\hline Causas obstétricas directas & 100.0 & 0.5 & 10.6 & 20.8 & 20.4 & 19.2 & 19.6 & 8.6 & 0.3 \\
\hline Hemorragia del embarazo y parto & 100.0 & 0.4 & 8.4 & 20.1 & 20.5 & 17.7 & 25.7 & 7.2 & 0.0 \\
\hline Toxemia del embarazo & 100.0 & 1.0 & 11.1 & 21.7 & 18.1 & 21.2 & 18.6 & 8.0 & 0.5 \\
\hline Infecciones del aparato genitourinario & 100.0 & 0.0 & 11.1 & 33.3 & 55.6 & 0.0 & 0.0 & 0.0 & 0.0 \\
\hline Parto obstruido & 100.0 & 0.0 & 0.0 & 15.4 & 15.4 & 30.8 & 23.1 & 15.4 & 0.0 \\
\hline Complicaciones del puerperio & 100.0 & 0.0 & 13.2 & 27.4 & 16.0 & 23.6 & 15.1 & 4.7 & 0.0 \\
\hline Las demás obstétricas directas & 100.0 & 0.3 & 11.2 & 18.0 & 23.9 & 16.5 & 18.0 & 11.8 & 0.3 \\
\hline Causas obstétricas indirectas & 100.0 & 2.2 & 15.6 & 20.0 & 17.8 & 26.7 & 15.6 & 2.2 & 0.0
\end{tabular}

* Razones por 10000 nacidos vivos registrados según edad de la madre

Fuente: Instituto N acional de Estadística, Geografía e Informática (IN EGI) y Dirección General de Estadística e Informática, SSA 


\section{Razón de mortalidad materna por causa Según entidad federativa. México, 1997}

\begin{tabular}{|c|c|c|c|c|c|c|c|c|c|c|c|}
\hline \multirow[b]{3}{*}{ Entidad } & \multicolumn{10}{|c|}{ Número de defunciones por causas obstétricas directas } & \multirow{3}{*}{$\begin{array}{c}\text { Causas obstétricas } \\
\text { indirectas/ } \\
\text { defunciones }\end{array}$} \\
\hline & \multicolumn{2}{|c|}{ Total } & \multirow{2}{*}{$\begin{array}{l}\text { Aborto/ } \\
\text { defun- } \\
\text { ciones }\end{array}$} & \multirow{2}{*}{$\begin{array}{c}\text { Obstétricas } \\
\text { directas. } \\
\text { Subtotal }\end{array}$} & \multirow{2}{*}{$\begin{array}{l}\text { Hemorragia } \\
\text { del embarazo } \\
\text { y parto }\end{array}$} & \multirow{2}{*}{ Toxemia } & \multirow{2}{*}{$\begin{array}{c}\text { Infección } \\
\text { genito } \\
\text { urinaria }\end{array}$} & \multirow{2}{*}{$\begin{array}{l}\text { Parto } \\
\text { obs- } \\
\text { truido }\end{array}$} & \multirow{2}{*}{$\begin{array}{l}\text { Compli- } \\
\text { cación del } \\
\text { puerperio }\end{array}$} & \multirow{2}{*}{$\begin{array}{c}\text { Las demás } \\
\text { obstétricas } \\
\text { directas }\end{array}$} & \\
\hline & $\begin{array}{l}\text { Defun } \\
\text { ciones }\end{array}$ & Razón* & & & & & & & & & \\
\hline Estados Unidos Mexicanos & 1266 & 4.7 & 107 & 1114 & 249 & 415 & 9 & 13 & 106 & 322 & 45 \\
\hline Aguascalientes & 8 & 3.2 & 1 & 7 & 1 & 4 & 0 & 0 & 0 & 2 & 0 \\
\hline Baja California & 11 & 1.9 & 2 & 9 & 3 & 5 & 0 & 0 & 0 & 1 & 0 \\
\hline Baja California Sur & 4 & 4.1 & 1 & 3 & 1 & 0 & 0 & 0 & 0 & 2 & 0 \\
\hline Campeche & 8 & 4.2 & 1 & 7 & 2 & 1 & 0 & 0 & 0 & 4 & 0 \\
\hline Coahuila & 12 & 2.1 & 0 & 11 & 3 & 5 & 0 & 0 & 0 & 3 & 1 \\
\hline Colima & 6 & 4.9 & 0 & 5 & 3 & 0 & 1 & 0 & 0 & 1 & 1 \\
\hline Chiapas & 83 & 6.3 & 3 & 80 & 19 & 16 & 0 & 2 & 11 & 32 & 0 \\
\hline Chihuahua & 38 & 4.9 & 0 & 37 & 2 & 22 & 0 & 1 & 1 & 11 & 1 \\
\hline Distrito Federal & 93 & 5.1 & 12 & 78 & 13 & 39 & 0 & 0 & 5 & 21 & 3 \\
\hline Durango & 15 & 3.1 & 0 & 15 & 1 & 5 & 0 & 0 & 3 & 6 & 0 \\
\hline Guanajuato & 48 & 3.5 & 4 & 41 & 7 & 12 & 0 & 1 & 4 & 17 & 3 \\
\hline Guerrero & 65 & 5.3 & 7 & 57 & 22 & 11 & 0 & 0 & 8 & 16 & 1 \\
\hline Hidalgo & 29 & 4.0 & 2 & 26 & 1 & 15 & 0 & 1 & 1 & 8 & 1 \\
\hline Jalisco & 70 & 4.3 & 3 & 61 & 9 & 24 & 3 & 0 & 9 & 16 & 6 \\
\hline México & 204 & 6.2 & 18 & 179 & 37 & 79 & 1 & 0 & 16 & 46 & 7 \\
\hline Michoacán & 54 & 4.2 & 6 & 48 & 17 & 12 & 2 & 0 & 5 & 12 & 0 \\
\hline Morelos & 20 & 5.2 & 3 & 17 & 5 & 5 & 0 & 0 & 3 & 4 & 0 \\
\hline$N$ ayarit & 13 & 5.0 & 1 & 11 & 1 & 3 & 0 & 0 & 2 & 5 & 1 \\
\hline N uevo León & 21 & 2.4 & 2 & 15 & 2 & 5 & 0 & 0 & 3 & 5 & 4 \\
\hline 0 axaca & 88 & 7.5 & 7 & 81 & 22 & 26 & 0 & 1 & 5 & 27 & 0 \\
\hline Puebla & 74 & 4.7 & 6 & 66 & 14 & 27 & 0 & 3 & 8 & 14 & 2 \\
\hline Q uerétaro & 18 & 4.7 & 0 & 18 & 5 & 7 & 0 & 0 & 2 & 4 & 0 \\
\hline Q uintana Roo & 7 & 3.2 & 2 & 5 & 3 & 2 & 0 & 0 & 0 & 0 & 0 \\
\hline San Luis Potosí & 31 & 4.7 & 3 & 27 & 9 & 10 & 0 & 0 & 3 & 5 & 1 \\
\hline Sinaloa & 16 & 2.0 & 1 & 13 & 1 & 7 & 0 & 0 & 0 & 5 & 2 \\
\hline Sonora & 17 & 3.0 & 1 & 13 & 2 & 8 & 0 & 0 & 1 & 2 & 3 \\
\hline Tabasco & 31 & 5.3 & 1 & 30 & 10 & 11 & 0 & 0 & 4 & 5 & 0 \\
\hline Tamaulipas & 23 & 3.4 & 1 & 16 & 2 & 8 & 0 & 0 & 2 & 4 & 6 \\
\hline Tlaxcala & 23 & 8.6 & 2 & 21 & 1 & 11 & 0 & 0 & 1 & 8 & 0 \\
\hline Veracruz & 89 & 4.2 & 11 & 76 & 23 & 21 & 1 & 3 & 6 & 22 & 2 \\
\hline Yucatán & 23 & 6.1 & 4 & 19 & 4 & 4 & 0 & 0 & 3 & 8 & 0 \\
\hline Zacatecas & 23 & 6.0 & 2 & 21 & 4 & 10 & 1 & 1 & 0 & 5 & 0 \\
\hline Extranjero & 1 & & 0 & 1 & 0 & 0 & 0 & 0 & 0 & 1 & 0 \\
\hline
\end{tabular}

* Razón por 10000 nacidos vivos registrados

Fuente: Instituto N acional de Estadística, Geografía e Informática (IN EGI) y Dirección General de Estadística e Informática, SSA 


\begin{tabular}{|c|c|c|c|c|}
\hline & $\begin{array}{l}\text { ORTALI } \\
\text { GUNAS }\end{array}$ & $\begin{array}{l}\text { uadro } \\
\text { MUJE } \\
\text { DE LA }\end{array}$ & $\begin{array}{l}\text { DS SEGÚ } \\
\text { co, } 199\end{array}$ & \\
\hline Variable & & & Defuncior & 49 años \\
\hline & No. & $\%$ & No. & $\%$ \\
\hline Sitio de la defunción & 1266 & 100.0 & 27089 & 100.0 \\
\hline Unidad Médica & 887 & 70.1 & 13372 & 49.4 \\
\hline Hogar & 283 & 22.4 & 10327 & 38.1 \\
\hline 0 tro sitio & 73 & 5.8 & 2502 & 9.2 \\
\hline No especificado & 23 & 1.8 & 888 & 3.3 \\
\hline Atención médica & 1266 & 100.0 & 27089 & 100.0 \\
\hline Sí & 967 & 76.4 & 20940 & 77.3 \\
\hline No & 277 & 21.9 & 4714 & 17.4 \\
\hline No especificado & 22 & 1.7 & 1435 & 5.3 \\
\hline Escolaridad & 1266 & 100.0 & 27089 & 100.0 \\
\hline Sin escolaridad & 221 & 17.5 & 4683 & 17.3 \\
\hline Menos de 3 años de primaria & 179 & 14.1 & 3726 & 13.8 \\
\hline 3 a 5 años de primaria & 169 & 13.3 & 3339 & 12.3 \\
\hline Primaria completa & 295 & 23.3 & 6501 & 24.0 \\
\hline Secundaria & 207 & 16.4 & 3985 & 14.7 \\
\hline Preparatoria & 98 & 7.7 & 2228 & 8.2 \\
\hline Profesional & 66 & 5.2 & 1585 & 5.9 \\
\hline No especificado & 31 & 2.4 & 1042 & 3.8 \\
\hline Derechohabiencia & 1266 & 100.0 & 27089 & 100.0 \\
\hline $\mathrm{N}$ inguna & 861 & 68.0 & 13746 & 50.7 \\
\hline IMSS & 240 & 19.0 & 8761 & 32.3 \\
\hline ISSSTE & 53 & 4.2 & 1362 & 5.0 \\
\hline Pemex & 3 & 0.2 & 140 & 0.5 \\
\hline Fuerzas armadas & 5 & 0.4 & 200 & 0.7 \\
\hline 0 tro & 19 & 1.5 & 513 & 1.9 \\
\hline Más de una & 1 & 0.1 & 43 & 0.2 \\
\hline No especificado & 84 & 6.6 & 2324 & 8.6 \\
\hline Estado civil & 1266 & 100.0 & 27089 & 100.0 \\
\hline Soltera & 145 & 11.5 & 7861 & 29.0 \\
\hline Casada & 765 & 60.4 & 13236 & 48.9 \\
\hline Unión libre & 314 & 24.8 & 3282 & 12.1 \\
\hline Separada & 7 & 0.6 & 604 & 2.2 \\
\hline Divorciada & 6 & 0.5 & 390 & 1.4 \\
\hline Viuda & 6 & 0.5 & 1134 & 4.2 \\
\hline No especificado & 23 & 1.8 & 582 & 2.1 \\
\hline Certificante de la defunción & 1266 & 100.0 & 27089 & 100.0 \\
\hline Médico tratante & 378 & 29.9 & 6945 & 25.6 \\
\hline 0 tro médico & 720 & 56.9 & 14223 & 52.5 \\
\hline Médico legista & 87 & 6.9 & 5034 & 18.6 \\
\hline No médico & 45 & 3.6 & 454 & 1.7 \\
\hline No especificado & 36 & 2.8 & 433 & 1.6 \\
\hline
\end{tabular}

IMSS: Instituto Mexicano del Seguro Social

ISSSTE: Instituto de Seguridad y Servicios Sociales de los Trabajadores del Estado Pemex: Petróleos Mexicanos

Fuente: Instituto N acional de Estadística, Geografía e Informática (IN EGI) y Dirección General de Estadística e Informática, SSA 


\section{Cuadro VI}

Mortalidad fetal y Perinatal. México, 1980-1997

\begin{tabular}{|c|c|c|c|c|c|c|c|c|c|c|c|c|}
\hline \multirow{4}{*}{ Año } & \multicolumn{9}{|c|}{ Fetal } & \multirow{3}{*}{\multicolumn{2}{|c|}{$\begin{array}{l}\text { Neonatal } \\
\text { temprana } \\
\text { ( }<7 \text { días) }\end{array}$}} & \multirow{4}{*}{$\begin{array}{c}\text { Perinatal } \\
\text { (28 s. y + } \\
\text { y }<7 \text { días) } \\
\text { Tasa }\end{array}$} \\
\hline & \multirow{2}{*}{\multicolumn{2}{|c|}{ Total }} & & & \multirow{2}{*}{\multicolumn{2}{|c|}{$20-27$}} & en sema & & & & & \\
\hline & & & \multicolumn{2}{|c|}{$<20$} & & & \multicolumn{2}{|c|}{$28 y+$} & \multirow{2}{*}{$\begin{array}{l}\text { No especificado } \\
\text { No. }\end{array}$} & & & \\
\hline & No. & Tasa & No. & Tasa & No. & Tasa & No. & Tasa & & No. & Tasa & \\
\hline 1980 & 32464 & 13.4 & 1759 & 0.7 & 5169 & 2.1 & 20902 & 8.6 & 4634 & 26266 & 10.8 & 19.4 \\
\hline 1981 & 31579 & 12.5 & 1532 & 0.6 & 4796 & 1.9 & 20216 & 8.0 & 5035 & 25934 & 10.2 & 18.2 \\
\hline 1982 & 28788 & 12.0 & 1511 & 0.6 & 4936 & 2.1 & 17881 & 7.5 & 4460 & 24502 & 10.2 & 17.7 \\
\hline 1983 & 28652 & 11.0 & 1354 & 0.5 & 5050 & 1.9 & 17891 & 6.9 & 4357 & 24475 & 9.4 & 16.3 \\
\hline 1984 & 28541 & 11.4 & 1653 & 0.7 & 5464 & 2.2 & 18345 & 7.3 & 3079 & 21689 & 8.6 & 15.9 \\
\hline 1985 & 21697 & 8.2 & 1272 & 0.5 & 4079 & 1.5 & 14779 & 5.6 & 1567 & 19564 & 7.4 & 13.0 \\
\hline 1986 & 21399 & 8.3 & 1137 & 0.4 & 3899 & 1.5 & 15151 & 5.9 & 1212 & 18874 & 7.3 & 13.2 \\
\hline 1987 & 21976 & 7.9 & 1133 & 0.4 & 4291 & 1.5 & 15515 & 5.6 & 1037 & 19242 & 6.9 & 12.5 \\
\hline 1988 & 27350 & 10.4 & 2036 & 0.8 & 5527 & 2.1 & 19033 & 7.3 & 754 & 20622 & 7.9 & 15.2 \\
\hline 1989 & 28877 & 11.0 & 2078 & 0.8 & 6032 & 2.3 & 20196 & 7.7 & 571 & 24078 & 9.2 & 16.9 \\
\hline 1990 & 29519 & 10.8 & 2074 & 0.8 & 6095 & 2.2 & 20908 & 7.6 & 442 & 24139 & 8.8 & 16.4 \\
\hline 1991 & 29456 & 10.7 & 2151 & 0.8 & 6216 & 2.3 & 20661 & 7.5 & 428 & 23202 & 8.4 & 15.9 \\
\hline 1992 & 29644 & 10.6 & 2340 & 0.8 & 6341 & 2.3 & 20728 & 7.4 & 235 & 22879 & 8.2 & 15.6 \\
\hline 1993 & 28772 & 10.1 & 2242 & 0.8 & 6250 & 2.2 & 20018 & 7.0 & 262 & 21711 & 7.6 & 14.6 \\
\hline 1994 & 28784 & 9.9 & 2420 & 0.8 & 6400 & 2.2 & 19707 & 6.8 & 207 & 21391 & 7.4 & 14.2 \\
\hline 1995 & 28201 & 9.6 & 2504 & 0.9 & 6546 & 2.4 & 18962 & 6.9 & 189 & 21052 & 7.7 & 14.5 \\
\hline 1996 & 27157 & 10.0 & 2724 & 1.0 & 6408 & 2.4 & 17854 & 6.6 & 171 & 19952 & 7.4 & 14.0 \\
\hline 1997 & 27135 & 10.1 & 2938 & 1.1 & 6762 & 2.5 & 17284 & 6.4 & 151 & 19809 & 7.3 & 13.7 \\
\hline
\end{tabular}

Tasa por 1000 nacidos vivos registrados

Fuente: Instituto N acional de Estadística, Geografía e Informática (IN EGI) y Dirección General de Estadística e Informática, SSA 


\section{Cuadro VII}

\section{Mortalidad fetal y Perinatal Según entidad federativa de ResidenCia habitual. México, 1997}

\begin{tabular}{|c|c|c|c|c|c|c|c|c|c|c|c|c|}
\hline \multirow{3}{*}{ Entidad } & \multicolumn{9}{|c|}{ Fetal } & \multirow{2}{*}{\multicolumn{2}{|c|}{$\begin{array}{l}\text { Neonatal } \\
\text { temprana } \\
\text { ( }<7 \text { días })\end{array}$}} & \multirow{3}{*}{$\begin{array}{c}\text { Perinatal } \\
\text { (28 s. y + } \\
\text { y <7 días) } \\
\text { Tasa }\end{array}$} \\
\hline & \multicolumn{2}{|c|}{ Total } & \multicolumn{2}{|c|}{$<20$} & \multicolumn{2}{|c|}{$\begin{array}{c}\text { Edad gestacio } \\
20-27\end{array}$} & \multicolumn{2}{|c|}{$28 y+$} & \multirow{2}{*}{$\begin{array}{c}\text { No especificado } \\
\text { No. }\end{array}$} & & & \\
\hline & No. & Tasa & No. & Tasa & No. & Tasa & No. & Tasa & & No. & Tasa & \\
\hline Estados Unidos Mexicanos & 27135 & 10.1 & 2938 & 1.1 & 6762 & 2.5 & 17284 & 6.4 & 151 & 19809 & 7.3 & 13.7 \\
\hline Aguascalientes & 290 & 11.7 & 10 & 0.4 & 69 & 2.8 & 210 & 8.5 & 1 & 230 & 9.3 & 17.8 \\
\hline Baja California & 691 & 12.2 & 99 & 1.7 & 207 & 3.6 & 379 & 6.7 & 6 & 569 & 10.0 & 16.7 \\
\hline Baja California Sur & 107 & 10.9 & 5 & 0.5 & 24 & 2.5 & 78 & 8.0 & 0 & 75 & 7.7 & 15.6 \\
\hline Campeche & 131 & 6.8 & 3 & 0.2 & 27 & 1.4 & 101 & 5.3 & 0 & 121 & 6.3 & 11.6 \\
\hline Coahuila & 355 & 6.2 & 35 & 0.6 & 95 & 1.7 & 222 & 3.9 & 3 & 300 & 5.3 & 9.2 \\
\hline Colima & 117 & 9.5 & 6 & 0.5 & 27 & 2.2 & 84 & 6.8 & 0 & 90 & 7.3 & 14.1 \\
\hline Chiapas & 1125 & 8.5 & 44 & 0.3 & 172 & 1.3 & 897 & 6.8 & 12 & 728 & 5.5 & 12.3 \\
\hline Chihuahua & 732 & 9.5 & 65 & 0.8 & 195 & 2.5 & 453 & 5.9 & 19 & 645 & 8.3 & 14.2 \\
\hline Distrito Federal & 2823 & 15.5 & 525 & 2.9 & 885 & 4.9 & 1408 & 7.7 & 5 & 1779 & 9.8 & 17.5 \\
\hline Durango & 254 & 5.3 & 2 & 0.0 & 47 & 1.0 & 202 & 4.2 & 3 & 97 & 2.0 & 6.2 \\
\hline Guanajuato & 2108 & 15.2 & 280 & 2.0 & 502 & 3.6 & 1324 & 9.6 & 2 & 1456 & 10.5 & 20.1 \\
\hline Guerrero & 321 & 2.6 & 1 & 0.0 & 59 & 0.5 & 259 & 2.1 & 2 & 345 & 2.8 & 4.9 \\
\hline Hidalgo & 660 & 9.2 & 70 & 1.0 & 165 & 2.3 & 425 & 5.9 & 0 & 428 & 6.0 & 11.9 \\
\hline Jalisco & 1945 & 11.9 & 323 & 2.0 & 506 & 3.1 & 1112 & 6.8 & 4 & 1414 & 8.6 & 15.4 \\
\hline México & 4926 & 15.0 & 861 & 2.6 & 1417 & 4.3 & 2641 & 8.1 & 7 & 3086 & 9.4 & 17.5 \\
\hline Michoacán & 966 & 7.6 & 73 & 0.6 & 209 & 1.6 & 679 & 5.3 & 5 & 665 & 5.2 & 10.5 \\
\hline Morelos & 413 & 10.8 & 21 & 0.6 & 111 & 2.9 & 279 & 7.3 & 2 & 299 & 7.8 & 15.1 \\
\hline $\mathrm{N}$ ayarit & 80 & 3.1 & 4 & 0.2 & 22 & 0.9 & 54 & 2.1 & 0 & 91 & 3.5 & 5.6 \\
\hline Nuevo León & 671 & 7.7 & 18 & 0.2 & 149 & 1.7 & 494 & 5.7 & 10 & 527 & 6.1 & 11.8 \\
\hline 0 axaca & 903 & 7.7 & 30 & 0.3 & 153 & 1.3 & 710 & 6.1 & 10 & 721 & 6.2 & 12.2 \\
\hline Puebla & 2140 & 13.6 & 120 & 0.8 & 578 & 3.7 & 1441 & 9.2 & 1 & 1779 & 11.3 & 20.5 \\
\hline Q uerétaro & 494 & 12.9 & 36 & 0.9 & 108 & 2.8 & 350 & 9.2 & 0 & 340 & 8.9 & 18.1 \\
\hline Q uintana Roo & 219 & 10.1 & 2 & 0.1 & 47 & 2.2 & 169 & 7.8 & 1 & 166 & 7.7 & 15.5 \\
\hline San Luis Potosí & 735 & 11.2 & 98 & 1.5 & 162 & 2.5 & 472 & 7.2 & 3 & 447 & 6.8 & 14.0 \\
\hline Sinaloa & 84 & 1.1 & 2 & 0.0 & 16 & 0.2 & 64 & 0.8 & 2 & 129 & 1.6 & 2.4 \\
\hline Sonora & 426 & 7.6 & 8 & 0.1 & 92 & 1.6 & 323 & 5.8 & 3 & 414 & 7.4 & 13.2 \\
\hline Tabasco & 438 & 7.5 & 3 & 0.1 & 74 & 1.3 & 354 & 6.0 & 7 & 408 & 7.0 & 13.0 \\
\hline Tamaulipas & 498 & 7.4 & 81 & 1.2 & 138 & 2.1 & 278 & 4.2 & 1 & 367 & 5.5 & 9.6 \\
\hline Tlaxcala & 312 & 11.7 & 13 & 0.5 & 81 & 3.0 & 214 & 8.0 & 4 & 303 & 11.3 & 19.3 \\
\hline Veracruz & 1495 & 7.0 & 88 & 0.4 & 291 & 1.4 & 1091 & 5.1 & 25 & 1154 & 5.4 & 10.5 \\
\hline Yucatán & 342 & 9.0 & 6 & 0.2 & 55 & 1.5 & 275 & 7.3 & 6 & 350 & 9.2 & 16.5 \\
\hline Zacatecas & 314 & 8.2 & 6 & 0.2 & 71 & 1.9 & 232 & 6.1 & 5 & 282 & 7.4 & 13.5 \\
\hline Extranjero & 20 & 19.6 & 0 & 0.0 & 8 & 7.8 & 10 & 9.8 & 2 & 4 & & 13.7 \\
\hline
\end{tabular}

Tasa por 1000 nacidos vivos registrados

Fuente: Instituto N acional de Estadística, Geografía e Informática (IN EGI) y Dirección General de Estadística e Informática, SSA 


\section{Cuadro VIII}

\section{MoRTALIDAd POR CÁNCER CERVICOUTERINO Y MAMARIO. MéXICO, 1980-1997}

\begin{tabular}{|c|c|c|c|c|c|c|c|c|}
\hline \multirow[b]{2}{*}{ Año } & \multicolumn{3}{|c|}{ Cervicouterino } & \multicolumn{3}{|c|}{ Mamario } & \multicolumn{2}{|c|}{ Total cervicouterino y mamario } \\
\hline & Defunciones & Tasa & Peso relativo & Defunciones & Tasa & Peso relativo & Defunciones & Peso relativo \\
\hline 1980 & 2543 & 16.9 & 17.4 & 1226 & 8.2 & 8.4 & 3769 & 25.8 \\
\hline 1981 & 2626 & 16.9 & 16.9 & 1333 & 8.6 & 8.6 & 3959 & 25.5 \\
\hline 1982 & 2960 & 18.3 & 18.3 & 1289 & 8.0 & 8.0 & 4249 & 26.3 \\
\hline 1983 & 3057 & 18.3 & 18.4 & 1428 & 8.5 & 8.6 & 4485 & 26.9 \\
\hline 1984 & 3304 & 19.0 & 19.0 & 1470 & 8.5 & 8.5 & 4774 & 27.5 \\
\hline 1985 & 3572 & 19.8 & 18.8 & 1672 & 9.3 & 8.8 & 5244 & 27.6 \\
\hline 1986 & 3621 & 19.4 & 18.6 & 1768 & 9.5 & 9.1 & 5389 & 27.7 \\
\hline 1987 & 3900 & 20.2 & 19.2 & 1988 & 10.3 & 9.8 & 5888 & 29.0 \\
\hline 1988 & 4096 & 20.4 & 19.5 & 2054 & 10.2 & 9.8 & 6150 & 29.3 \\
\hline 1989 & 4290 & 20.6 & 20.0 & 2165 & 10.4 & 10.1 & 6455 & 30.0 \\
\hline 1990 & 4280 & 20.2 & 19.8 & 2230 & 10.5 & 10.3 & 6510 & 30.1 \\
\hline 1991 & 4194 & 19.2 & 18.9 & 2379 & 10.9 & 10.7 & 6573 & 29.6 \\
\hline 1992 & 4346 & 19.2 & 18.7 & 2559 & 11.3 & 11.0 & 6905 & 29.8 \\
\hline 1993 & 4369 & 18.7 & 18.3 & 2718 & 11.6 & 11.4 & 7087 & 29.8 \\
\hline 1994 & 4365 & 18.1 & 18.0 & 2785 & 11.5 & 11.5 & 7150 & 29.5 \\
\hline 1995 & 4392 & 17.6 & 17.4 & 3026 & 12.1 & 12.0 & 7418 & 29.4 \\
\hline 1996 & 4526 & 17.6 & 17.2 & 3106 & 12.1 & 11.8 & 7632 & 29.0 \\
\hline 1997 & 4534 & 17.1 & 16.9 & 3220 & 12.2 & 12.0 & 7754 & 29.0 \\
\hline
\end{tabular}

Tasa por 100000 mujeres de 20 años y más

Peso relativo: porcentaje respecto al total de defunciones por tumores malignos en mujeres

Fuente: Instituto N acional de Estadística, Geografía e Informática (IN EGI) y Dirección General de Estadística e Informática, SSA 


\section{Cuadro IX}

Mortalidad POR CÁNCER CerVicouterino y Mamario. MéXico, 1997

\begin{tabular}{|c|c|c|c|c|c|c|c|c|}
\hline \multirow[b]{2}{*}{ Entidad } & \multicolumn{3}{|c|}{ Cervicouterino } & \multicolumn{3}{|c|}{ Mama } & \multicolumn{2}{|c|}{ Total cervicouterino y mamario } \\
\hline & Defunciones & Tasa & Peso relativo & Defunciones & Tasa & Peso relativo & Defunciones & Peso relativo \\
\hline Estados Unidos Mexicanos & 4534 & 17.1 & 16.9 & 3220 & 12.2 & 12.0 & 7754 & 29.0 \\
\hline Aguascalientes & 32 & 13.0 & 12.5 & 42 & 17.0 & 16.3 & 74 & 28.8 \\
\hline Baja California & 100 & 15.1 & 17.2 & 67 & 10.1 & 11.5 & 167 & 28.6 \\
\hline Baja California Sur & 14 & 12.3 & 12.7 & 14 & 12.3 & 12.7 & 28 & 25.5 \\
\hline Campeche & 40 & 22.9 & 23.5 & 16 & 9.2 & 9.4 & 56 & 32.9 \\
\hline Coahuila & 90 & 13.8 & 13.7 & 98 & 15.0 & 14.9 & 188 & 28.6 \\
\hline Colima & 33 & 23.8 & 19.5 & 24 & 17.3 & 14.2 & 57 & 33.7 \\
\hline Chiapas & 204 & 21.4 & 23.0 & 63 & 6.6 & 7.1 & 267 & 30.1 \\
\hline Chihuahua & 154 & 18.2 & 16.2 & 104 & 12.3 & 11.0 & 258 & 27.2 \\
\hline Distrito Federal & 363 & 13.2 & 10.6 & 558 & 20.2 & 16.2 & 921 & 26.8 \\
\hline Durango & 54 & 14.0 & 14.7 & 40 & 10.3 & 10.9 & 94 & 25.5 \\
\hline Guanajuato & 171 & 13.8 & 15.1 & 132 & 10.6 & 11.7 & 303 & 26.8 \\
\hline Guerrero & 146 & 19.6 & 22.6 & 64 & 8.6 & 9.9 & 210 & 32.5 \\
\hline Hidalgo & 79 & 13.6 & 15.0 & 55 & 9.4 & 10.5 & 134 & 25.5 \\
\hline Jalisco & 290 & 16.4 & 14.0 & 288 & 16.3 & 13.9 & 578 & 27.9 \\
\hline México & 449 & 12.8 & 16.4 & 330 & 9.4 & 12.1 & 779 & 28.5 \\
\hline Michoacán & 238 & 23.2 & 21.2 & 112 & 10.9 & 10.0 & 350 & 31.2 \\
\hline Morelos & 97 & 23.4 & 20.9 & 37 & 8.9 & 8.0 & 134 & 28.8 \\
\hline $\mathrm{N}$ ayarit & 64 & 26.3 & 21.5 & 26 & 10.7 & 8.8 & 90 & 30.3 \\
\hline Nuevo León & 132 & 12.1 & 12.1 & 198 & 18.2 & 18.2 & 330 & 30.4 \\
\hline 0 axaca & 221 & 24.6 & 22.9 & 69 & 7.7 & 7.2 & 290 & 30.1 \\
\hline Puebla & 234 & 17.9 & 18.8 & 114 & 8.7 & 9.2 & 348 & 28.0 \\
\hline Q uerétaro & 50 & 14.6 & 18.3 & 37 & 10.8 & 13.6 & 87 & 31.9 \\
\hline Q uintana Roo & 26 & 13.8 & 27.1 & 7 & 3.7 & 7.3 & 33 & 34.4 \\
\hline San Luis Potosí & 119 & 19.5 & 19.1 & 46 & 7.5 & 7.4 & 165 & 26.5 \\
\hline Sinaloa & 103 & 15.2 & 15.6 & 93 & 13.7 & 14.1 & 196 & 29.7 \\
\hline Sonora & 98 & 16.0 & 15.1 & 107 & 17.5 & 16.5 & 205 & 31.6 \\
\hline Tabasco & 108 & 23.3 & 24.9 & 36 & 7.8 & 8.3 & 144 & 33.2 \\
\hline Tamaulipas & 151 & 19.3 & 18.8 & 125 & 16.0 & 15.5 & 276 & 34.3 \\
\hline Tlaxcala & 50 & 20.0 & 21.4 & 27 & 10.8 & 11.5 & 77 & 32.9 \\
\hline Veracruz & 451 & 22.4 & 21.5 & 176 & 8.7 & 8.4 & 627 & 29.9 \\
\hline Yucatán & 114 & 25.7 & 21.9 & 53 & 12.0 & 10.2 & 167 & 32.1 \\
\hline Zacatecas & 45 & 12.6 & 11.7 & 38 & 10.6 & 9.9 & 83 & 21.6 \\
\hline Extranjero & 14 & & 9.9 & 24 & & 16.9 & 38 & 26.8 \\
\hline
\end{tabular}

Tasa por 100000 mujeres de 20 años y más

Peso relativo: porcentaje respecto al total de tumores malignos en mujeres

Fuente: Instituto N acional de Estadística, Geografía e Informática (IN EGI) y Dirección General de Estadística e Informática, SSA 